\title{
Amodal completion as a basis for illusory contours
}

\author{
R. H. DAY and R. T. KASPERCZYK \\ Monash University, Clayton, Victoria, Australia
}

\begin{abstract}
Kanizsa's (1979) explanation of illusory contours in terms of amodal completion of perceptually incomplete elements was examined in five experiments by varying the elements partly delineating the corners and sides of a rectangle. The results from magnitude estimation of contour strength showed that (1) incomplete corner elements consistently resulted in stronger illusory contours than did complete elements, although contours nevertheless always occurred with the latter, (2) contours were always stronger with side elements than without but were not always reliably different in strength when the side elements were parts of an incomplete form, (3) incomplete octagons and slightly different but complete elements resulted in contours of the same strength, (4) the larger of two incomplete corner elements resulted in stronger contours, and (5) incomplete irregular elements resulted in stronger contours than did complete irregular ones, although weak contours again occurred with the latter. Together, the data do not strongly support the view that perceptual completion of incomplete figural elements is the basis of illusory contours. An alternative interpretation in terms of the spreading of induced contrast to partially delineated borders is proposed. It is suggested that apparent overlay occurring independently of perceptual completion enhances these borders and so strengthens the illusory contours.
\end{abstract}

Kanizsa (1979) invoked the two configurations shown here in Figures $1 A$ and $1 B$ in support of his explanation of illusory contours. He argued that in the configuration shown in Figure $1 \mathrm{~A}$, consisting of four incomplete octagons and an incomplete cross formed from four lines, the inner white rectangle splits from the rest of the background and an illusory border consequently forms around it. In the case of the configuration shown in Figure 1B, consisting of four complete and "self-sufficient" crosses and four similarly complete and self-sufficient diamonds, the whole background remains unitary and no rectangle with an illusory border emerges. Kanizsa (1979) then went on to argue that the basis of apparent stratification in Figure 1A and its nonoccurrence in Figure 1B is the respective incompleteness and completeness of the elements that make up the figures. When the incomplete elements are completed in perception, they acquire a greater measure of "stability" and "compactness." Consequently, the borders of the inner right-angle sectors in the octagons appear to belong to an enclosed rectangle. This necessitates the rectangle appearing to overlay the octagons and cross and thus appearing to occlude parts of both. Since the rectangle seems to be in front of the perceptually complete elements,

Support for this research was provided by a grant from the Australian Research Grants Committee. The assistance of Judy Sack and Vladimir Kohout in the preparation of stimulus materials is gratefully acknowledged. Requests for reprints should be addressed to R. H. Day, Department of Psychology, Monash University, Clayton, Victoria 3168, Australia.
A

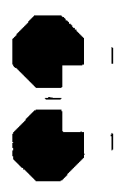

Figure 1. Kanizsa's ceptual incompleteness (A), completeness (B), and reduced area of corner elements (C) on illusory contours.

it appears to be detached from the background and bounded by contours. On the other hand, the black crosses and diamonds are perceived as complete, and therefore the borders of the innermost right angles of the crosses are perceived as belonging to the crosses themselves, not to a rectangle. For this reason, no apparent stratification occurs and no rectangle or illusory edges emerge.

Thus, according to Kanizsa $(1974,1976,1979)$, illusory contours have their origin in perceptually incomplete elements. Completion of these elements in perception requires the formation of an apparently opaque shape lying in front of them. This in turn requires the formation of contours. Because the apparently complete elements in figures such as Figure 1A have a phenomenal rather than a sensory or "modal" presence, they are called by Kanizsa "amodal": hence the term "amodal completion."

Kanizsa's $(1974,1976,1979)$ explanation can be challenged on at least two grounds: its reliance almost entirely on casual, nonquantitative evidence, 
and the exclusion of induced contrast effects as primary determinants of the contours. Since the five experiments described here were derived from these criticisms, it is useful to examine the criticisms in more detail.

First, while informal observation leaves little doubt that illusory contours are present in Figure 1A, their absence entirely in Figure $1 B$ is questionable. Without formal quantitative data, this question is not easily resolved. The same criticism applies to a number of figures about which Kanizsa (1979) made strong assertions concerning the presence or absence of illusory contours.

Second, Kanizsa $(1974,1976,1979)$ treated the altered brightness of the apparently occluding form (the partially delineated rectangle in Figure 1) as a consequence rather than as a cause of amodal completion. He claimed that the region acquires an "object-character" with greater surface density and solidity. Thus, like the illusory contours, the altered brightness was attributed to apparent stratification of the figure following amodal completion. Against this view, there is now a substantial body of data to suggest that illusory contours are due in some part to induced differences in brightness (Brigner \& Gallagher, 1974; Day \& Jory, 1978, 1980; Frisby \& Clatworthy, 1975; Jory \& Day, 1979; Kennedy, 1979; Redies \& Spillman, 1981; Ronchi \& Mori, 1959; Spillman, Fuld, \& Gerrits, 1976). It is known that the area of inducing elements affects the degree of brightness contrast (Diamond, 1955). It is therefore conceivable that the more prominent illusory contours in Figure 1A derive from the contrast being stronger than in Figure 1B. It is to be noted, however, that there is evidence for the occurrence of illusory contours in the absence of induced contrast (Parks, 1980).

Kanizsa $(1976,1979)$ ruled out the effect of area by reference to Figure $1 \mathrm{C}$. He pointed out that the incomplete elements in this figure, although half the area of those in Figure 1B, nevertheless give rise to prominent illusory contours. No comparison was made between the strength of the contours in Figures $1 \mathrm{~A}$ and 1C. Presumably, no difference would be expected, since amodal completion of incomplete elements occurs in both. However, it is to be noted that Kanizsa (1976) conceded the possibility that although brightness contrast is not necessary for the formation of illusory contours, it could nevertheless play a role.

The general aim of the five experiments reported here was quantitatively to examine, using magnitude estimation, the amodal completion explanation of illusory contours. The purpose of the first experiment was to ascertain whether illusory contours occur in Figure 1B with complete elements and, if so, their strength relative to those in Figure $1 \mathrm{~A}$ with incomplete elements. To assess the relative contributions of the elements delineating the corners (octagons and crosses) and those impinging on the sides (lines and diamonds) of the rectangle, the combinations of these "corner" and "side" elements were systematically varied. The second and third experiments were designed to establish whether illusory contours occur with complete, self-sufficient elements differing from the incomplete octagons only by the removal of an additional right-angle sector. In the fourth experiment, the strength of illusory contours in Figure 1C was compared with that of those in Figures $1 \mathrm{~A}$ and $1 \mathrm{~B}$. The purpose of the last experiment was to establish whether a contour-strength difference similar to that between Figures $1 \mathrm{~A}$ and $1 \mathrm{~B}$ extends to figures made up of markedly different complete and incomplete elements. The effects of element and figure symmetry were also investigated in this experiment.

It is emphasized that the five experiments were concerned with the validity of Kanizsa's (1974, $1976,1979)$ amodal completion explanation of illusory contours, not with a comparison between this explanation and that couched in terms of contrast (Day \& Jory, 1978). However, an attempt to account for the data in terms of contrast and its spreading to partially delineated borders is included in the final discussion.

\section{METHOD}

The measurement of illusory contour strength poses a number of problems of measurement. There seems to be no exact physical counterpart of contour strength into which the perceptual judgment can be translated as is the case with illusions of size, brightness, and velocity. Neither variations in luminance on either side of a real contour nor variations along the sharpnessblurredness continuum seem to reflect the changes in strength that occur. And it is not appropriate to require subjects to judge the shape or brightness of the bounded region; the shape is clearly defined by fragments of real contour, as can be seen in Figures 1-4. In any case, Brussell, Stober, and Bodinger (1977) and Jory (1979) have pointed out that brightness and contour strength can vary independently.

Following preliminary experiments, the most satisfactory method for the measurement of illusory contour strength has proved to be magnitude estimation (D'Amato, 1970; Stevens, 1956). It can be noted that although this technique has been shown to lack discriminative power in some (but not all) situations (Coren \& Girgus, 1972), it has proved effective and reliable in establishing the effect on illusory contour strength of minimal differences in luminance (Jory \& Day, 1979) and of illumination level and retinal size (Dumais \& Bradley, 1976).

Magnitude estimation was used in the five experiments reported here with illusory contour strength in the well-known Kanizsa triangle as the standard. The method has proved entirely successful.

\footnotetext{
Subjects

Undergraduate and graduate students, mainly the former, served as subjects and were paid for their services. Groups of either 12 or 24 subjects, with more or less equal representation of men and women, took part in each experiment. A few of
} 
these in Experiment 1 were familiar with illusory contours, but none in Experiments 2-5 were.

\section{Apparatus and Stimulus Figures}

The subjects sat at a table and viewed the stimulus figures from about $570 \mathrm{~mm}$. There was no head restraint. The stimulus figures were drawn in black about the centers of white cards. The $250 \times 250 \mathrm{~mm}$ cards (Swedish pasteboard No. 8) were placed on a rack tilted back at $135 \mathrm{deg}$ so that they were frontoparallel and approximately at right angles to the line of regard. The card with the standard figure was placed on the right, and the cards with the figures to be rated were placed on the left. Illumination was provided by a single incandescent source. The luminance of the white regions was $13.75 \mathrm{~cd} / \mathrm{m}^{2}$.

The standard was the well-known Kanizsa triangle figure (Kanizsa, 1955) shown in Figure 2. This figure was chosen because preliminary observations had shown that the illusory contours along the partially delineated borders are confidently and quickly perceived by almost all observers. The incomplete black circular elements at the corners of this figure were $30 \mathrm{~mm}$ in diameter and the edges of the 60-deg sectors were $14 \mathrm{~mm}$ along an edge. The lines of the 60-deg angles forming parts of an equilateral triangle were $28 \mathrm{~mm}$ long. The sides of the white partially enclosed triangle and those of the outline triangle were both $80 \mathrm{~mm}$ long.

The figures whose illusory contours were rated relative to those of the standard are described and illustrated in the appropriate sections below.

\section{Procedure}

The subjects were first shown the illusory contours in the standard figure. No attempt was made to disguise the illusory nature of the contours. The subjects were simply shown the contours along the borders of the standard triangle and along those of another figure. All subjects readily perceived the illusory effect. The subjects were then instructed in rating the strength of the contours. They were told that, in making their estimates, they should treat the contours of the standard as strength 10 , that the complete absence of illusory contours should always be rated 0 , and that ratings of greater and less than 10 were entirely acceptable. The importance of estimating the strength of the contours themselves rather than the vividness of the bounded shape was stressed. No subject expressed any difficulty in estimating the strength of the illusory contours relative to those of the standard.

The stimulus cards were presented in two series, with a different random order for each. During the interval of half a minute or so between each series, the subjects were reminded of the instructions. They were also told not to try to remember and repeat the values assigned in the first series but to assign whatever values now seemed appropriate.

\section{EXPERIMENT 1}

Kanizsa (1979) asserts that, whereas clearly visible illusory contours occur in Figure 1A, which consists of incomplete corner octagons and lines forming an incomplete cross, they do not occur in Figure 1B, which consists of perceptually complete corner crosses and outlined diamonds. Without quantitative data to back these claims, two questions can be posed. First, are illusory contours entirely absent in Figure 1B? Some informal preliminary observations suggested that, although weak, they nevertheless occur. Second, do the incomplete octagons and the parts of the incomplete cross impinging on the hypothetical edge of the rectangle contribute equally to the formation of the illusory contours? It is conceivable that, as Day and Jory (1980) suggested, the ends of the lines merely determine the location of the contours. The purpose of the first experiment was to answer these two questions. For convenience, the octagons and crosses defining the corners of the white rectangle will be called corner elements, and the lines and diamonds impinging on the edges of the rectangle, side elements.

\section{Subjects and Stimulus Figures}

A total of 24 subjects, 14 women and 10 men, took part in the experiment.

The eight stimulus figures, along with the standard Kanizsa figure, are shown in Figure 2 . Since the figures were viewed from about $570 \mathrm{~mm}, 10 \mathrm{~mm}$ subtended a visual angle of about $1 \mathrm{deg}$. The inner right-angle sectors in the black crosses and octagons delineated the corners of a $40 \times 60 \mathrm{~mm}$ white rectangle. The arms of the crosses were $40 \mathrm{~mm}$ long and $14 \mathrm{~mm}$ wide. The edges delineating the corners of the rectangle were also $14 \mathrm{~mm}$. The incomplete octagons were constructed by filling in, up to a diagonal edge, three of the four angles in the cross. For this reason, alternating sides of the octagon were unequal in length. They were 14 and $18 \mathrm{~mm}$ long. The distance between parallel sides was $40 \mathrm{~mm}$. In Figures $2 \mathrm{~A}$ and $2 \mathrm{~B}$, there were no side elements. In Figures $2 \mathrm{C}$ and $2 \mathrm{D}$, lines $11 \mathrm{~mm}$ long and $.6 \mathrm{~mm}$ thick were drawn at right angles to the hypothetical border of the

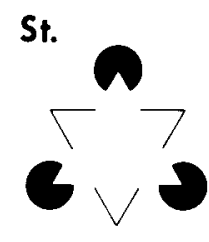

A

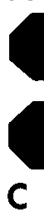

C

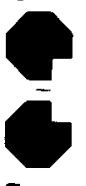

E

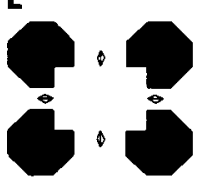

G
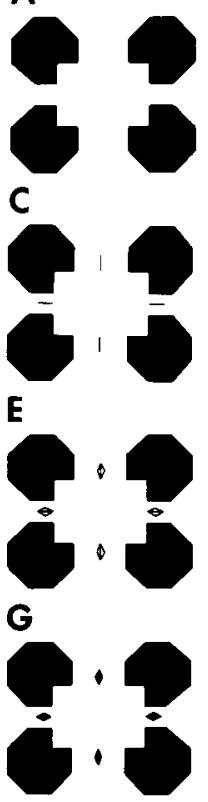

B

$\mathbf{F}$

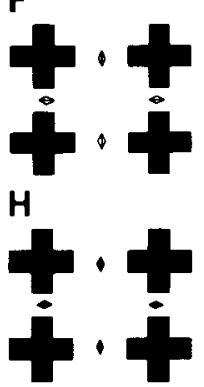

Figure 2. The figures, varying in completeness and incompleteness of corner and side elements, used in Experiment 1. St. refers to the standard (the Kanizsa triangle) in terms of whose illusory contours the strength of those in the other figures was established. 
rectangle, with one end of each line located precisely on the border midway between the corners. In Figures $2 \mathrm{E}$ and $2 \mathrm{~F}$, diamond-shaped outlines $5 \mathrm{~mm}$ wide were drawn symmetrically about the 11-mm-long lines. In Figures $2 \mathrm{G}$ and $2 \mathrm{H}$, the diamonds were filled in to form black shapes. This was done to eliminate the central lines in Kanizsa's diamond shapes. It was conceivable that these lines could be perceptually isolated and thus still be perceived as parts of an incomplete cross.

In terms of amodal completion, it was expected that the figures with incomplete elements would give rise to stronger illusory contours than those consisting of complete elements. It was also expected that the strongest contours would occur when both corner and side elements were incomplete and that no contours would be perceived when both were complete and selfsufficient.

\section{Results and Discussion}

Mean estimates of illusory contour strength are shown with their standard deviations in Table 1. It can be seen that the means for the figures formed from the octagons are consistently greater than for those formed from the crosses and that the means for the figures with side elements, either lines or diamonds, are greater than those in the comparable figures without them. Casual inspection shows also that illusory contour strength with the lines (Figures $2 \mathrm{C}$ and $2 \mathrm{D}$ ) was slightly greater than with the open or closed diamonds (Figures 2E, 2F, 2G, and 2H).

The first two observations were confirmed in a two-way analysis of variance in which the main factors were the corners (2) and side elements (4). Both factors proved to be significant $[F(1,23)=34.08$, $p<.001$, and $F(3,69)=8.73, p<.001$, respectively]. The interaction between the two was not $(F<1)$. The observation that illusory contours were stronger in the figures that had side elements consisting of lines was not confirmed by a series of NewmanKeuls tests. Separate tests for the octagon and cross figures showed that, while contour strength for fig- ures with side elements was invariably significantly greater than for those without ( $p<.01$ for all comparisons), there were no differences between estimates for the different types of side elements $(p>$ .05 for all comparisons). Since the mean for the figure consisting of four crosses (Figure 2B) was relatively small, the precaution was taken to test whether this and other means were different from zero effect. A series of $t$ tests showed that all means significantly exceeded zero at $p<.001$.

Thus, although the strength of illusory contours was consistently greater with the incomplete octagon elements than with the complete crosses, illusory contours invariably occurred with the latter. Indeed, inspection of the 48 scores for Figure $2 F$, in which all the elements were complete, revealed that there were only two zero estimates. Furthermore, there was no statistically reliable difference between open or filled-in diamonds complete in themselves and lines that could be construed as parts of a partially occluded cross. However, contour strength was consistently greater with side elements than without. This outcome must be interpreted cautiously. It is conceivable, as always with "no-difference" outcomes, that a larger sample might have revealed a significant effect of incomplete side elements. This issue is considered again in the general discussion. Finally, the absence of any differences between figures with filled-in and open diamonds indicates that the lines forming long axes in the open diamonds did not themselves uniquely contribute to the formation of the contours.

\section{EXPERIMENT 2}

Jory (1979) pointed out that a relatively slight alteration of the incomplete octagons in Figure 1A

Table 1

Mean Estimates of Contour Strength and Their Standard Deviations for the Five Experiments

\begin{tabular}{|c|c|c|c|c|c|c|c|c|c|c|c|c|}
\hline & \multicolumn{12}{|c|}{ Stimulus Figure } \\
\hline & $\mathbf{A}$ & B & $\mathrm{C}$ & D & $\mathbf{E}$ & $\mathbf{F}$ & G & $\mathbf{H}$ & I & $\mathbf{J}$ & $\mathbf{K}$ & $\mathbf{L}$ \\
\hline \multicolumn{13}{|c|}{ Experiment 1} \\
\hline $\begin{array}{l}\text { Mean } \\
\text { SD }\end{array}$ & $\begin{array}{l}5.17 \\
3.39\end{array}$ & $\begin{array}{l}2.83 \\
2.69\end{array}$ & $\begin{array}{l}7.54 \\
2.63\end{array}$ & $\begin{array}{l}5.04 \\
2.13\end{array}$ & $\begin{array}{l}6.98 \\
4.50\end{array}$ & $\begin{array}{l}4.21 \\
2.52\end{array}$ & $\begin{array}{l}6.94 \\
3.84\end{array}$ & $\begin{array}{l}4.48 \\
3.36\end{array}$ & & & & \\
\hline \multicolumn{13}{|c|}{ Experiment 2} \\
\hline $\begin{array}{l}\text { Mean } \\
\text { SD }\end{array}$ & $\begin{array}{l}6.19 \\
4.21\end{array}$ & $\begin{array}{l}4.27 \\
3.70\end{array}$ & $\begin{array}{l}6.50 \\
3.84\end{array}$ & $\begin{array}{r}10.58 \\
4.30\end{array}$ & $\begin{array}{l}8.78 \\
4.80\end{array}$ & $\begin{array}{r}10.90 \\
4.98\end{array}$ & $\begin{array}{l}9.52 \\
4.37\end{array}$ & $\begin{array}{l}6.50 \\
3.43\end{array}$ & $\begin{array}{l}8.75 \\
3.78\end{array}$ & $\begin{array}{l}9.19 \\
4.37\end{array}$ & $\begin{array}{l}7.00 \\
3.68\end{array}$ & $\begin{array}{l}8.89 \\
3.98\end{array}$ \\
\hline \multicolumn{13}{|c|}{ Experiment 3} \\
\hline $\begin{array}{l}\text { Mean } \\
\text { SD }\end{array}$ & $\begin{array}{l}6.29 \\
5.47\end{array}$ & & $\begin{array}{l}9.54 \\
4.82\end{array}$ & $\begin{array}{l}9.75 \\
4.56\end{array}$ & & $\begin{array}{r}11.79 \\
4.97\end{array}$ & $\begin{array}{l}9.13 \\
3.63\end{array}$ & & $\begin{array}{l}9.88 \\
4.25\end{array}$ & $\begin{array}{l}8.63 \\
3.97\end{array}$ & & $\begin{array}{r}11.00 \\
4.37\end{array}$ \\
\hline \multicolumn{13}{|c|}{ Experiment 4} \\
\hline $\begin{array}{l}\text { Mean } \\
\text { SD }\end{array}$ & $\begin{array}{l}9.50 \\
3.19\end{array}$ & $\begin{array}{l}5.00 \\
2.15\end{array}$ & $\begin{array}{l}7.46 \\
4.20\end{array}$ & & & & & & & & & \\
\hline \multicolumn{13}{|c|}{ Experiment 5} \\
\hline $\begin{array}{l}\text { Mean } \\
\text { SD }\end{array}$ & $\begin{array}{l}6.54 \\
2.80\end{array}$ & $\begin{array}{l}9.77 \\
2.42\end{array}$ & $\begin{array}{l}6.73 \\
2.77\end{array}$ & $\begin{array}{l}9.75 \\
2.46\end{array}$ & $\begin{array}{l}5.50 \\
2.92\end{array}$ & $\begin{array}{l}9.94 \\
2.86\end{array}$ & & & & & & \\
\hline
\end{tabular}


renders the elements symmetrical and complete but that illusory contours seem nevertheless to persist. Jory's modified elements and the figures formed by them are shown in Figure 3. The modification involves the removal of a second right-angle sector opposite to that of the first, rendering the elements bilaterally symmetrical. They now appear as two side-by-side elongated hexagons. It can be noted that, as Jory suggested, the alteration gives the appearance of symmetry and completeness to the elements.

If Jory's (1979) observations were to be sustained, Kanizsa's $(1974,1976,1979)$ explanation in terms of amodal completion of incomplete structures would be called into question. The purpose of the second experiment, therefore, was to compare the strength of illusory contours in figures having Jory's "double hexagon" elements (as they will be called for convenience) with that in figures having incomplete octagons and complete crosses as in Experiment 1.

Convincing evidence against an interpretation of illusory contours in terms of amodal completion of figural elements has already been reported by Kennedy (1978) and Rock and Anson (1979). Kennedy (1978) demonstrated that the contours occur in line figures that are complete in themselves, and Rock and Anson (1979) demonstrated that they are present in figures composed of irregular but perceptually complete corner elements. However, in neither of these studies was the strength of illusory contours in figures composed of perceptually complete and incomplete elements compared. This was the main point of the second experiment.

Since the same subjects served under all conditions, it was conceivable that the double-hexagon elements would be perceived as incomplete by association with the incomplete octagons. Experiment 3, which involved independent groups, served as a control for this possibility.

\section{Subjects and Stimulus Patterns}

There were 24 subjects, 13 women and 11 men, none of whom had participated earlier.

The 12 stimulus figures are shown in Figure 3. The dimensions of the incomplete octagons, the crosses, and the partially enclosed rectangle were the same as in Experiment 1. The dimensions of the new elements were the same as the octagons and differed only by the removal of a second right-angle sector identical to the first.

The side elements were intended to suggest more strongly an incomplete cross and four self-sufficient diamonds and wedges. They were identical in length $(17 \mathrm{~mm})$ and area $\left(34 \mathrm{~mm}^{2}\right)$. The lines were $2 \mathrm{~mm}$ wide, and the diamonds and wedges were $4 \mathrm{~mm}$ wide at their greatest widths.

In terms of completeness and incompleteness of elements, it was expected that illusory contour strength in the figures with double hexagons would approximate more closely that in the figures with crosses than that in the figures with incomplete octagons.

\section{Results and Discussion}

Mean estimates of contour strength and their standard deviations are shown in Table 1. Inspection suggests that the strength of illusory contours in the double-hexagon figures is more or less the same as that in the octagon figures and notably greater than that in the cross figures. Again, it appears that the means for figures with side elements are, on the whole, greater than those without them and the means for side elements in the form of lines are slightly greater than those in the form of elongated diamonds or wedges.

A two-way analysis of variance, in which the main factors were corner and side elements, and a series of Newman-Keuls tests confirmed the first two impressions. The third was partially confirmed. The effects of both corner and side elements were significant $[F(2,23)=23.77, p<.001$, and $F(3,69)=$ $23.17, \mathrm{p}<.001$, respectively]. The interaction between the two factors was not significant $(F<1)$. Newman-Keuls tests showed that the means for both the incomplete-octagon and double-hexagon figures were significantly different from the mean for the cross figure ( $p<.01$ in each case) but not from one another ( $p>.05$ in each case). The means for figures with side elements proved significantly greater than for those without them. This was so for figures consisting of double hexagons, incomplete octa-

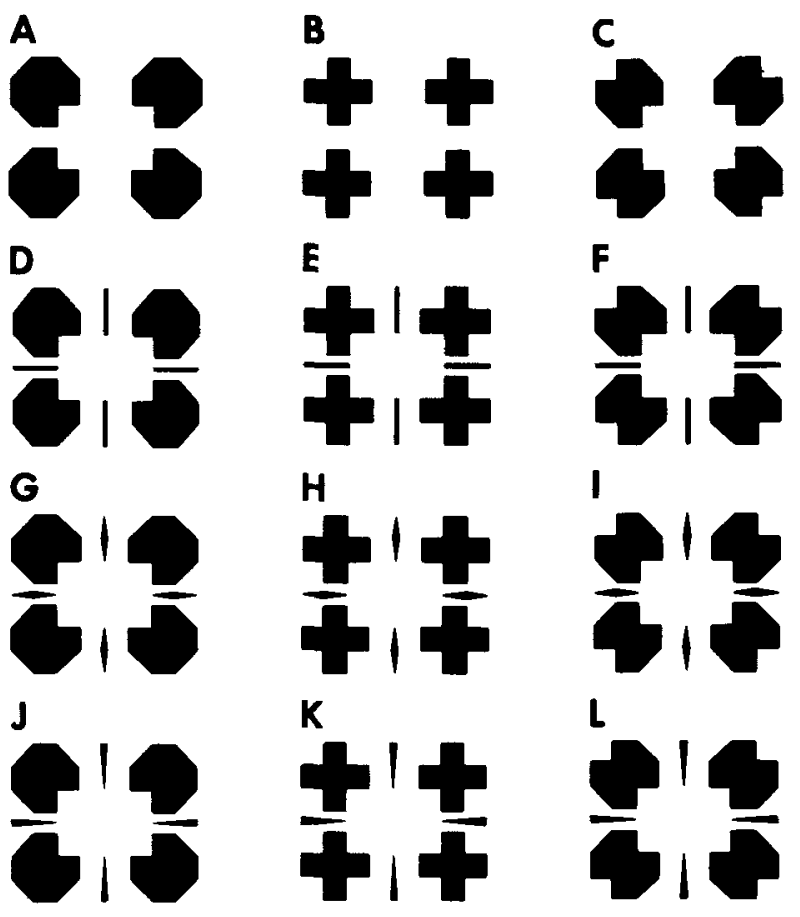

Figure 3. Figures used in Experiments 2 and 3. The "donblebexagon" elements (C, F, I, and L) differ from the "incompleteoctagon" (A, D, G, and J) elements only by the absence of an additional right-angle sector. 
gons, and crosses. In the cases of figures with crosses and double hexagons, the means of those with line side elements (Figures 3E and 3F) were significantly greater than those with either diamonds or wedges (Figures $3 \mathrm{H}$ and $3 \mathrm{I}$ and Figures $3 \mathrm{~K}$ and $3 \mathrm{~L}$ ). However, there was no difference between the means for the three types of side elements in the figures with octagons. Thus, there is weak support for perceptual completion of the incomplete cross in the double-hexagon and cross figures.

Even if it were argued that the double-hexagon elements are not as perceptually complete and as self-sufficient as the crosses due to, inter alia, the greater familiarity of the latter, it would hardly be disputed that the former are more so than the incomplete octagons. Therefore, the absence of any difference in illusory contour strength between the figures with double hexagons and those with incomplete octagons questions the involvement of amodal completion.

In this experiment, side elements in the form of lines that together made up an incomplete cross did give rise to slightly stronger illusory contours in the case of those figures with crosses and double hexagons at the corners. This was not so for figures with incomplete octagons.

Finally, it is of interest to note that not only was the mean (10.90) for one double-hexagon figure (Figure 3F) about the same (10.58) as that for the incomplete-octagon figure with the same side elements (Figure 3D), but also it was greater than that for the standard. In other words, the illusory contours in Figure 3F were, on the average, stronger than those in the Kanizsa triangle figure. This result adds support to the finding that perceptual completion of incomplete elements is not essential for the formation of illusory contours.

\section{EXPERIMENT 3}

The double-hexagon elements in Experiment 2 differed from the incomplete-octagon elements only in the removal of a second right-angle sector. However, the difference in the appearance of the elements was marked (Figure 3). In Kanizsa's terms, the double-hexagon elements appear to be balanced and complete and the octagon shapes unbalanced and incomplete. However, it is conceivable that, since both elements were derived from an octagon and were presented to the same subjects, the double hexagons could also have been perceived as incomplete. That is to say, the subjects might have perceived the double hexagons as incomplete through association with the incomplete octagons to which they were also exposed. Were this so, the absence of any difference between the strength of the illusory contours in the two figures could reasonably be attributed to the perceived incompleteness of the elements composing each.
The purpose of Experiment 3 was to examine this issue more closely. Figures composed of double hexagons and incomplete octagons were presented to separate groups. Thus, the experiment served as a control for the possibility that double-hexagon elements might have been perceived as incomplete as a result of their association with incomplete octagons.

\section{Subjects and Stimulus Figures}

There were two groups of 12 subjects, one consisting of 7 women and 5 men and the other of 6 women and 6 men. The first group judged the strength of illusory contours in the four figures with incomplete-octagon elements (Figures 3A, 3D, 3G, and $3 \mathrm{~J}$ ), and the second judged their strength in the four figures with double-hexagon elements (Figures 3C, 3F, 3I, and 3L).

\section{Results and Discussion}

Mean estimates of contour strength and their standard deviations are shown for the eight figures in Table 1. Preliminary inspection again suggested that there was no difference between the strength of illusory contours with octagons and those with double hexagons, that there was a weaker effect with those lacking side elements, and that there were slightly stronger illusory contours with side elements in the form of lines than in the form of diamonds or wedges. An analysis of variance in which side elements were a repeated factor and corner elements a between-subjects factor again confirmed the first and second impressions. The third impression was only partially confirmed. The difference between the means for the two corner elements was not significant $[F(1,22)=1.44, p>.05]$, whereas that between side elements was $[F(3,66)=33.76, p<.001]$. The interaction between the two factors also failed to achieve significance $[F(3,66)=2.20, p>.05]$. For the octagon figures, Newman-Keuls tests showed that, as earlier, the means for the figures with side elements were all greater than the mean for the figure without side elements. However, in the case of the double-hexagon figures, the results are not consistent with those of Experiment 2. The mean for the figure without side elements was less than only that with line elements. Furthermore, the mean for the latter figure proved to be significantly different from that for the figure with diamonds but not from that for the figure with wedges.

Thus, the outcomes of this experiment were essentially the same as those for Experiment 2 in regard to corner elements but not in regard to side elements. However, it can be concluded that the perceived completeness (or incompleteness) of the double-hexagon elements was not significantly influenced by the presentation of incomplete octagons in Experiment 2.

\section{EXPERIMENT 4}

Given that simultaneous brightness contrast varies directly with the area of the inducing stimulus 
(Diamond, 1955), it follows that the stronger illusory contours in figures with incomplete octagons (Figure 1A) than in those with complete crosses (Figure 1B) might be due in part to a greater effect of contrast. Kanizsa (1979) ruled out this possibility with Figure 1C, in which the corner elements are half the area of the crosses. As is clear from inspection, the illusory contours seem relatively stronger in this "half-cross" figure than in that made up of complete crosses. If, as Kanizsa $(1976,1979)$ contended, incompleteness is the basis of illusory contours, no difference in contour strength between Figures 1A and 1C would be expected unless, of course, the degree of apparent incompleteness itself varies between the two. Kanizsa $(1976,1979)$ did not entertain this latter possibility but implied that both are more or less equally complete.

Given the marked difference in area between Figures $1 \mathrm{~A}$ and $1 \mathrm{C}$, the absence of any difference in the strength of illusory contours would support an explanation in terms of amodal completion. A difference, however, would indicate that simultaneous contrast, apparent incompleteness of corner elements, or both are involved. It should be emphasized that the occurrence of a difference in contour strength would implicate apparent incompleteness as much as it would implicate contrast, since the former might also vary with the different elements.

Whether or not differences occur in the strength of illusory contours for the incomplete-octagon figure and for the half-cross figure was investigated in the fourth experiment. The cross figure was also included for comparison.

\section{Subjects and Stimulus Figures \\ There were 12 new subjects, 8 women and 4 men. \\ The three stimulus figures are shown in Figure 1. The dimen- sions of Figures $1 \mathrm{~A}$ and $\mathrm{BB}$ were the same as those in the pre- ceding experiments. The area of the corner elements in Figure 1C was exactly haif that of the crosses. The corner elements were constructed simply by dividing the crosses across a diagonal extent. The lines forming the side elements were the same in length and thickness $(11 \times .6 \mathrm{~mm})$ as in the earlier experiments.}

\section{Results and Discussion}

It can be seen from Table 1, in which the mean estimates of contour strength and their standard deviations are shown, that the mean for the figure with half-cross elements is about midway between the means for the other two figures. A one-way analysis of variance showed that the effect of corner elements was significant $[F(2,11)=12.67, p<.001]$. Newman-Keuls tests showed that the differences in rated contour strength between any pair of figures was significant at $p<.01$.

The results of this experiment do not favor an interpretation of illusory contours in terms of simultaneous brightness contrast any more than they do one in terms of apparent incompleteness of corner elements. If contrast were the only factor involved, then the greatest effect would be expected with the elements of greatest area-the octagons-and the smallest with those of least area-the half crosses. This was not so. If apparent incompleteness as an invariant quality were the only factor, then the strength of the contours with the incomplete octagons and half crosses would be the same. A significant difference in contour strength occurred between these two elements. If apparent incompleteness were the only factor, then it would have to be conceived of as varying in degree between the incomplete octagons and half crosses. A resolution of this issue is suggested in the general discussion below.

\section{EXPERIMENT 5}

At this point, two questions can be posed. First, do illusory contours occur in figures with complete, self-sufficient elements quite different in form and structure from crosses? An answer to this question would serve to indicate whether the occurrence of illusory contours in figures with complete elements is a general phenomenon. Second, since illusory contours varying in strength occurred in all the figures so far included, is there some common structural property that might be associated with their occurrence? Experiment 5 was concerned with these two questions.

To answer the first question, two sets of elements with irregular outlines were devised. For one set, the missing right-angle sector delineating the corner of the enclosed white rectangle was one of a number and therefore, as far as could be judged, did not lend an appearance of incompleteness to the elements. For the other set, the missing sector was the only such indentation and lent a strong appearance of incompleteness to the elements. The elements are shown in Figure 4.

In Experiments 1-4, the figures and the elements from which they were formed were all bilaterally symmetrical about one or more axes. For example, the cross elements were symmetrical about four axes, the incomplete octagon about one, and the figures formed by them about two. It therefore seemed possible that element or figure symmetry (or both), which was common throughout, is implicated in the formation of illusory contours. Accordingly, both symmetrical and asymmetrical elements were either complete or incomplete as described above.

\section{Subjects and Stimulus Figures}

There were 24 subjects, 16 women and 8 men. None had participated in earlier experiments in the series.

The corner elements in Figures $4 \mathrm{~A}, \mathbf{4 C}$, and $\mathbf{4 E}$ were irregular and angular in outline. Because the right-angle sector delineating a corner of the rectangle was not unique, the elements did not 

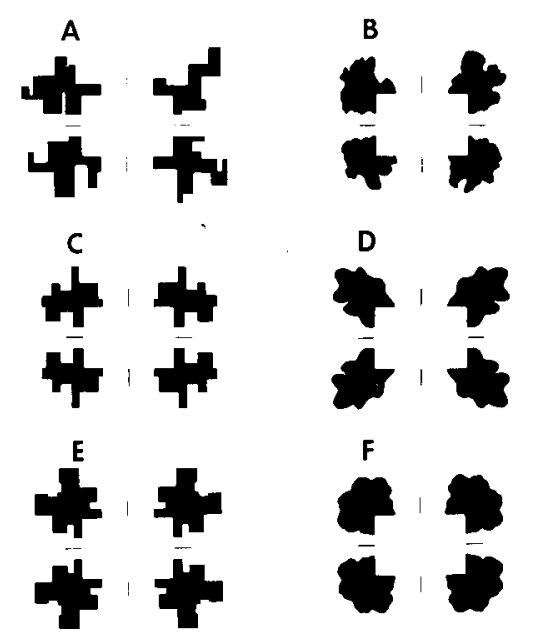

Figure 4. Irregular-outline complete $(\mathrm{A}, \mathrm{C}, \mathrm{E})$ and incomplete $(B, D, F)$ figures used in Experiment 5.

appear to be incomplete. The elements at the corners of Figures $4 \mathrm{~B}, 4 \mathrm{D}$, and $4 \mathrm{~F}$ were irregular and rounded in outline. The missing right-angle sector delineating a corner was in this case unique and lent an appearance of incompleteness to the elements.

The figures were in three pairs, of which one member was composed of complete elements and the other of incomplete elements. In Figures 4A and 4B, both the corner elements and figures themselves were asymmetrical, in Figures $4 \mathrm{C}$ and $4 \mathrm{D}$, the elements were asymmetrical and the figures were symmetrical, and in Figures $4 \mathrm{E}$ and $4 \mathrm{~F}$, both the elements and the figures were symmetrical. Although these three conditions did not exhaust the combinations of element and figure symmetry and asymmetry, they were considered sufficient to reveal if symmetry is a major determinant in the formation of illusory contours. The corner elements were between about $700-900 \mathrm{~mm}^{2}$, and the lines forming the side elements were $11 \mathrm{~mm}$ long and $.6 \mathrm{~mm}$ thick.

\section{Results and Discussion}

Mean contour strengths and standard deviations for the six figures are shown in Table 1 . It can be seen that, as earlier, contour strength for the three figures with complete corner elements (Figures 4A, $4 C$, and $4 E$ ) was consistently less than that for the figures with incomplete elements (Figures 4B, 4D, and $4 \mathrm{~F}$ ). No clear pattern of scores is immediately discernible for the three conditions of symmetry. A two-way analysis of variance involving types of corner element (2) and conditions of symmetry (3) showed that, whereas the former was a significant factor $[F(1,23)=90.34, p<.001]$, the latter was not $[F(2,46)=3.11, p>.05]$. However, the interaction between the two factors was significant $[F(2,46)$ $=3.23, \mathrm{p}<.05$ ]. Newman-Keuls tests showed that, although there were no differences between the means for the figures with complete elements, there were differences between those for the figures with incomplete ones. The means for Figures $4 \mathrm{~A}$ and $4 \mathrm{C}$ were both different from the mean for Figure $4 \mathrm{E}$. There was no difference between the means for Figures $4 \mathrm{~A}$ and $4 \mathrm{C}$. That is to say, illusory contours in the figure in which both the figure itself and the corner elements were symmetrical were weaker than in the figures in which both were asymmetrical and the figure alone was symmetrical.

In summary, as was the case in the earlier experiments with octagon and half-cross elements, the incomplete elements resulted in significantly stronger contours than did the complete elements. Nevertheless, as earlier, illusory contours occurred in the figures with complete elements. The results show also that in the case of the figures with angularoutline elements, bilateral symmetry of both elements and figure give rise to weaker contours than do either asymmetry of both or asymmetry of elements and symmetry of figure.

\section{GENERAL DISCUSSION}

The outcomes of the five experiments are entirely consistent in showing that illusory contours are stronger in figures in which the corners of the apparently contoured form-in this case a rectangleare delineated by elements that appear incomplete. This finding, which obtained with Kanizsa's elements (Figure 1) and with irregular elements (Figure 4), accords well with Kanizsa's (1974, 1976, 1979) explanation of illusory contours in terms of amodal completion of incomplete elements. Against this, however, there are two equally robust outcomes that are clearly not in accord with the amodal completion explanation. First, although illusory contours were invariably weaker with complete corner elements, they nevertheless persistently occurred regardless of whether or not side elements were present. It can, of course, be argued that the occurrence of the contours with the perceptually complete figure elements might be an artifact of magnitude estimation; the subjects might have been reluctant to assign zero estimates when no contours were visible. However, it will be recalled that the subjects were strongly encouraged throughout to assign 0 to those figures in which illusory contours could not be seen. It seems unlikely that consistent regression to the mean would occur with such strong and frequent emphasis on assigning zero estimates when no contours were visible. It could also be argued that subjects instructed to estimate the strength of illusory contours might do so in figures composed of complete corner and side elements simply because physical edges and points partially delineate an enclosed form. Kanizsa (1979) drew attention to the difference between mere delineation of an edge and the formation of a true illusory contour. This possibility can again be rebutted in terms of the strong emphasis on assigning 0 to figures in which no illusory contours were perceived. Given that the subjects were so instructed and were also shown 
examples of a strong contour in Kanizsa's triangle figure, it seems unlikely that they would confuse illusory contours with delineated edges.

Second, in Experiments 2 and 3, the illusory contours with apparently complete double-hexagon elements (Figure 3) were as strong as those with incomplete octagons. Even if it were contended that the double hexagons are perceptually less complete than the crosses, the finding that the contours are no different in strength from the contours with the incomplete octagons would indicate that the completion process is not critical for the formation of illusory contours. It might also be suggested that the consistent occurrence of illusory contours in figures with complete elements is an artifact of the procedure wherein subjects estimated contour strength for all figures in an experiment. Thus, it could be argued that a complete element such as a cross or a double hexagon might be perceived as incomplete through association with, say, an incomplete octagon. This seemed quite possible in Experiment 2, in which incomplete octagons and complete double-hexagon elements differed only by the removal from the latter of a second right-angle sector. However, the results of Experiment 3, in which independent groups estimated the strength of illusory contours in figures consisting only of one type of element, were the same as those in Experiment 2 . It therefore seems unlikely that judgment of one figure was unduly affected by association with another.

The results for side elements in the form of lines making up an incomplete cross compared with those for side elements in the form of diamonds and wedges each complete in itself are by no means clear cut. In Experiment 1, in which the side elements were similar to those in Kanizsa's $(1976,1979)$ figures, there was no difference between lines and open or closed diamonds. In Experiments 2 and 3, in which the elements were longer, there was a tendency for the contours to be stronger with lines than with diamonds or wedges. However, this was evident only for the figures in which the corners were defined by crosses or double hexagons. The tendency did not obtain for the octagon figures. Thus, at most, the evidence for completion of side elements as a determinant of illusory contour strength is weak. Whether or not lines forming an incomplete cross affect illusory contour strength depends in some part on their lengths and on the forms of the corner elements. Further investigation is clearly necessary.

It is reasonable to conclude from these experiments that Kanizsa's amodal completion explanation of illusory contours is not entirely adequate. At the same time, the data give notice that an alternative explanation must take into account that the contours occur with varying strength for all the corner ele- ments included in the experiments, that they are consistently stronger with incomplete than with complete corner elements, and that they are stronger with than without side elements regardless of whether these are or are not parts of an incomplete form. An alternative explanation must also encompass the now substantial and impressive body of evidence for the involvement of various forms of brightness contrast in the formation of illusory contours (see Halpern, 1981).

A clue to a more satisfactory explanation is given by inspection of the configurations illustrated in Figures 3-5. Regardless of the incompleteness or otherwise of corner elements, all the figures seem to convey an impression of a white rectangle overlaying parts of the corner elements. This impression varies in strength. It seems to be weak with the four crosses alone (Figure 2B), stronger with the four incomplete octagons alone (Figure 2A), and stronger when side elements such as lines or diamonds are added to either (Figures 2D, 2F, and $2 \mathrm{H}$ and Figures $2 \mathrm{C}, 2 \mathrm{E}$, and $2 \mathrm{G}$ ). Although these observations are entirely impressionistic, they do suggest an alternative basis for the formation of illusory contours.

It is suggested that "classical" or simultaneous contrast from the corner elements and line-end contrast generated by the ends of the side elements (Day \& Jory, 1978; Frisby \& Clatworthy, 1975; Kennedy, 1979) spread to the partially delineated borders of the rectangle (or other shapes) in a manner described earlier by Day and Jory (1980). It is suggested also that apparent overlay serves primarily to enhance the delineation of the border with which the illusory contour is coincident, by apparent stratification of the rectangular figure and the corner elements. That is to say, apparent overlay renders borders more prominent by separating the rectangle from the ground. The more salient the border, the stronger is the illusory contour.

It is emphasized that this interpretation of the effects of overlay in terms of the salience of partially delineated borders is speculative and does not follow immediately from the data reported. The intention in putting it forward is to provide a basis for further experiments on the role of apparent overlay in the formation of illusory contours. It is recognized also that the validity of this view rests on measurement of the strength or salience of apparent overlay, which is conceived of as a factor in variations in the strength of illusory contours.

Three final points are useful. First, Halpern (1981) showed by means of correlational and multipleregression methods that apparent depth, induced brightness, and figural features are implicated in the formation of illusory contours. More recently, Halpern, Salzman, Harrison, and Widaman (in press) used factor analysis to show that at least four 
factors, simultaneous contrast, line-end contrast, stimulus information for depth, and figural form, are involved. These analyses can be regarded as supportive of the thesis that is outlined here, that is, that figural features and apparent depth are involved in the enhancement of the partially defined edges along which illusory contours form. Second, overlay is only one source of stimulus information for depth. It is conceivable that since edges occur in depth as well as in two-dimensional arrays, other sources of depth information might serve to enhance partially defined edges and thereby strengthen illusory contours. The final point is in the nature of a reflection. There is an elegant economy about the formation of illusory contours as conceived of here. The stimulus elements that induce contrast also partially delineate the edges along which the contours form. If they also convey information for the edges of objects in depth, the contours are made stronger. It is as if the visual perceptual system has as one of its functions the completion and strengthening of object boundaries that are partially or inadequately delineated. The greater the level of contrast induced by the delineating elements, the stronger the boundaries are.

\section{REFERENCES}

Brigner, W., \& Gallagher, M. Subjective contours: Apparent depth or simultaneous brightness contrast? Perceptual \& Motor Skills, 1974, 38, 1047-1053.

Brusselt, E. M., Stober, S. R., \& Bodinger, D. M. Sensory information and subjective contour. American Journal of Psychology, 1977, 90, 145-156.

Coren, S., \& Girqus, J. S. A comparison of five methods of illusion measurement. Behavioral Research Methods \& Instrumentation, 1972, 4, 240-244.

D'Aмато, M. R. Experimental psychology: Methodology, psychophysics and learning. New York: McGraw-Hill, 1970.

DAY, R. H., \& JoRY, M. K. Subjective contours, visual acuity and line contrast. In J. C. Armington, J. E. Krauskopf, \& B. R. Wooten (Eds.), Visual psychophysics: Its physiological basis. New York: Academic Press, 1978.

DAY, R. H., \& JorY, M. K. A note on a second stage in the formation of illusory contours. Perception \& Psychophysics, $1980,27,89-91$.

Diamond, A. L. Foveal simultaneous contrast as a function of inducing field area. Journal of Experimental Psychology, 1955, 50, 144-152.

Dumais, S. T., \& Bradley, D. R. The effect of illumination level and retinal size on the apparent strength of subjective contours. Perception \& Psychophysics, 1976, 19, 339-345.

Frisby, J. P., \& Clatworthy, J. L. Illusory contours: Curious cases of simultaneous brightness contrast? Perception, 1975, 4, 349-357.

Halpern, D. F. The determinants of illusory-contour perception. Perception, 1981, 10, 199-213.

Halpern, D. F., Salzman, B., Harrison, W., \& Widaman, K. The multiple determination of illusory contours: 2. An empirical investigation. Perception, in press.

JoRY, M. K. The relationship between brightness and illusory contours. Unpublished doctoral thesis, Monash University, 1979.

JoRY, M. K., \& DAY, R. H. The relationship between brightness contrast and illusory contours. Perception, 1979, 8, 3-9.

KAnizsA, G. Margini quasi-percettivi in campi con stimolazione omogenea. Revista di Psicologia, 1955, 49, 7-30.

KANIZSA, G. Contours without gradients or cognitive contours. Italian Journal of Psychology, 1974, 1, 93-112.

KanizsA, G. Subjective contours. Scientific American, 1976, 234, 48-52.

Kanizsa, G. Organization in vision. New York: Praeger, 1979.

Kennedy, J. M. Illusory contours not due to completion. Perception, 1978, 7, 187-189.

KENNEDY, J. M. Subjective contours, contrast, and assimilation. In C. F. Nodine \& D. F. Fisher (Eds.), Perception and pictorial representation. New York: Praeger, 1979.

Parks, T. E. Subjective figures: Some unusual concomitant brightness effects. Perception, 1980, 9, 239-241.

Redies, C., \& Spillman, L. The neon color effect in the Ehrenstein illusion. Perception, 1981, 10, 667-681.

Rock, I., \& Anson, R. Illusory contours as the solution to a problem. Perception, 1979, 8, 665-681.

Ronchi, L., \& Mori, G. F. On the factors which affect the contrast enhancement in a figure with "quasi perceptive contours" and a practical application of such a figure. Atti Della Fondzione Girgio Ronchi, 1959, 14, 495-508.

Spillman, L., Fuld, K., \& Gerrits, H. J. M. Brightness contrast in the Ehrenstein illusion. Vision Research, 1976, 16, 713-719.

Strevens, S. S. The direct estimation of sensory magnitudesloudness. American Journal of Psychology, 1956, 69, 1-25.

(Manuscript received August 16, 1982;

revision accepted for publication December 15, 1982.) 